\title{
Caso clínico: Fístula Carótido Cavernosa
}

\section{Case report: Cavernous Carotid Fistula}

\author{
Marisa Arcos ${ }^{1 *}$, Guido Andres Toral Sanchez ${ }^{2}$ y Juan Sebastián Peña Aguilera ${ }^{2}$ \\ ${ }^{1}$ Universidad del Salvador, Argentina \\ 2 Universidad Católica de Cuenca \\ *marisaarcos@hotmail.com
}

DOI: https://doi.org/10.26871/killkana_salud.v3i1.103

\begin{abstract}
Resumen
Objetivo: Describir caso clínico sobre fístula carótida cavernosa para dar a conocer la importancia del diagnóstico precoz de la misma, así como del control de la presión intraocular. Materiales y Métodos: Reporte de un caso clínico de un paciente en el periodo de julio de 2017 apoyado en recopilación bibliográfica actualizada sobre el tema previamente descrito. Resultados: La fístula carótido cavernosa es una entidad poco frecuente en la patología neuroquirurgica, con gran repercusión Neuro - Oftalmológica, por lo cual es de importancia establecer cuál la clasificación, prevención, manejo óptimo y en qué pacientes está indicado realizar una intervención quirúrgica o no. ${ }^{1}$ La fístula carótido-cavernosa es una conexión anómala entre la arteria carótida y el seno cavernoso, puede ser espontánea en $25 \%$ de los casos o adquirida en el $75 \%$ restante, principalmente de origen traumático, en otros de los casos por mal formaciones arterio-venosas. ${ }^{2}$ En cuanto a la evolución de las fístulas carótido- cavernosas indirectas es variable, aproximadamente poseen una resolución espontánea, debido a la retrombosis del segmento afectado del seno cavernoso. Sin embargo, si ocurre pérdida progresiva de la visión y glaucoma intratable se requiere el manejo quirúrgico inmediato. Conclusiones: La fístula carótido-cavernosa es un reto en cuanto al diagnóstico, tratamiento, y complicaciones, ya que forman parte de las afecciones neuro-oftalmicas poco frecuentes, pues asi se puede obtener un pronóstico positivo y mantener la calidad de vida del paciente; el diagnóstico precoz es un punto clave.
\end{abstract}

Palabras clave: fístula Carótido Cavernosa, Malformación Arterio - Venosa, Glaucoma.

\begin{abstract}
Objective: To describe a case report of Carotid cavernous Fistula in order to highlight the importance of its early diagnosis and timely monitoring of intraocular pressure. Materials and Methods: Case report of a patient during July 2017, backed by an up to date bibliographic compilation on the previously described condition. Results: Cavernous-carotid fistula is an uncommon neurosurgical pathology, with a great impact in the field of Neuro-Ophthalmology. Thus, it is essential to describe its classification, prevention, optimal treatment and to determine which patients have, and which do not have surgical treatment indications. The carotid-cavernous fistula is an abnormal connection between the carotid artery and the cavernous sinus; $25 \%$ of cases can be spontaneous and the remaining $75 \%$ can be acquired, mainly of traumatic origin; or due to arteriovenous malformations. The evolution of indirect carotid-cavernous fistulas is varying; ranging from spontaneous relief, due to a newly formed thrombus in the affected region of the cavernous sinus. To progressive vision loss and untreatable glaucoma, which requires immediate surgical treatment. Conclusions: The diagnosis, treatment, and complications of Carotid cavernous fistula are challenging as it is an uncommon neuro-ophthalmological condition. Early diagnosis is the key to obtain a positive prognosis and maintain the patient's quality of life.
\end{abstract}

Key words: Cavernous Carotid Fistula, Malformation or arterio - venous, Glaucoma.

\section{Introducción}

La fístula carótido cavernosa es una comunicación anómala arterio-venosa poco frecuente entre la arteria carótida y el seno cavernoso a través de los canales intradurales de la arteria carótida interna o externa producida en la mayoría de los casos por traumatismos. ${ }^{3}$

Puede cursas daño ocular grave ya que la sangre dentro de las venas se arteriolizan y la presión intravenosa se eleva de manera que altera la dirección y velocidad de las mismas. ${ }^{3}$

Barrow describió una clasificación basada en criterios angiográficos y la dividió en 4 grupos, que son:

- A: Shunt directo entre arteria carótida interna y el seno intracavernoso. (Directa)

- B: Fístula dural procedente de arteria carótida interna. (Indirecta)

- C: Fístula alimentada por arteria carótida externa. (Indirecta) 
- D: Alimentada por ambas arterias carótidas. (Indirecta) $)^{3}$

Estudios han demostrado que también se producen por causas secundarias a procedimientos quirúrgicos, por ejemplo, angioplastias carotideas.

Las de origen espontáneos son de etiología desconocida, se pueden dar de manera indirecta a diversos factores predisponentes como Hipertensión Arterial(HTA), maniobras de valsalva, embarazo, etcétera.

Se ha visto una estrecha relación entre Fístula Carótido Cavernosa (FCC) y colagenopatias como ateroesclerosis, osteogénesis imperfecta entre otras.

Los síntomas y signos que se presentan son edema de párpados, soplo orbital, hiperemia, dolor orbital, exoftalmos pulsátil, pérdida de la agudeza visual, quemosis, dilatación pupilar, presión intraocular elevada, oftalmoplejía y diplopía

Puede presentar síntomas extraoculares tales como parálisis del nervio craneal, tinnitus, epistaxis, soplo intracraneal, y hemorragia intracraneal. ${ }^{4}$

La exoftalmia pulsátil se encuentra en el $90 \%$, asociada con una disminución de la agudeza visual. ${ }^{5}$

En la auscultación de la región peri orbitaria se puede evidenciar un soplo intracraneal sistólico-diastólico que desaparece con la compresión manual de la arteria carótida ipsilateral a nivel del cuello. ${ }^{5}$

La ecografía con Doppler color permite que la fístula se confirme con una sensibilidad elevada al mostrar una señal Doppler invertida en las venas oftálmicas dirigida a la cara sistólica. ${ }^{6}$

La Tomografía Axial Computarizada(TAC) busca signos indirectos, aumento del tamaño del seno cavernoso y de la vena oftálmica superior, infiltración de los músculos oculomotor y los tejidos orbitarios. ${ }^{6}$

La arteriografía cerebral es el Gold estándar para el diagnóstico de fístula carótido cavernosa, y para la implementación de la terapéutica en el mismo tiempo de intervención. ${ }^{6}$

Estudios aconsejan su realización desde el principio cuando se evidencia un exoftalmos pulsátil más un soplo orbital sistólico-diastólico. $\left({ }^{6}\right)$

La embolización transvenosa es un tratamiento muy efectivo en fístulas indirectas, requiere acceso al seno cavernoso a través del seno petroso inferior o a través de acceso quirúrgico a la vena oftálmica superior o punción transorbital directa a través de la fisura orbital superior. ${ }^{7}$

Los abordajes orbitarios se consideran arriesgados en comparación con el abordaje tradicional a través del seno petroso inferior, por lo que se utilizan de segunda opción. ${ }^{8}$

La disfunción del tronco encefálico con congestión venosa o hemorragia es una complicación poco frecuente de FCC. ${ }^{9}$ En la mayoría de las ocasiones, es causada por la congestión venosa.

La congestión venosa normalmente involucra el parénquima pontino, el mesencéfalo, el bulbo raquídeo o el cerebelo. $\left({ }^{9}\right)$
La presencia de hemorragia externa ya sea por epistaxis, drenaje venoso cortical o demostración angiográfica de una bolsa venosa, se han descrito como indicadores de un alto riesgo de mortalidad que requiere una intervención urgente. ${ }^{9,10}$

El pronóstico ha mejorado de forma considerable en los últimos 20 años gracias a los avances en la neurorradiología intervencionista. $^{8}$

\section{Caso Clinico}

Paciente de género masculino de 53 años de edad, mestizo, y casado. Con antecedentes de hipertensión diagnosticada hace 5 años, tratado con Losartan $50 \mathrm{mg} /$ día, tabaquismo crónico hace aproximadamente 35 años. Sin antecedentes quirúrgicos ni traumatismos craneales previos. Paciente refiere que desde hace aproximadamente seis meses y sin causa aparente presenta dolor tipo pulsátil de 3/10 en Escala Visual Analógica del Dolor (EVA) a nivel de globo ocular izquierdo de manera intermitente, una o dos veces por semana, que cede con administración de analgésicos (automedicados que no recuerda el nombre). El cual en el último mes el dolor se intensifica a 6/10 en EVA acompañado de quemosis e irritación ocular. Al cuadro se suma movimientos oculares restringidos y leve pérdida de la agudeza visual, razón por lo cual acude consulta.

Al momento de la consulta se evidenció un Tensión Arterial (TA): 150/80 mmHg, frecuencia cardiaca de 101 latidos por minuto y temperatura axilar de $37.4{ }^{\circ} \mathrm{C}$.

$\mathrm{Al}$ examen físico se encontró con fascies álgicas, un Indice de Masa Corporal (IMC) 31 con lo que se evidencia una Obesidad Grado 1, presencia de Exoftalmia izquierda, quemosis conjuntival, Soplo Ocular audible izquierdo, movimientos oculares restringidos. Párpado izquierdo edematizado, inflamación conjuntival, presencia de vasos episclerales (cabeza de medusa). Hipertensión Ocular $32 \mathrm{mmHg}$, disminución de la agudeza visual ojo izquierdo, con test de Snellen 20/70. Paciente se encontró vigil, orientado en tiempo espacio y persona, sin signos de focalidad neurológica.

Se realizó exámenes de sangre sin encontrar ninguna alteración. También se realizó Tomografía Axial Computarizada en la cual se encontró dilatación de la vena oftálmica posterior izquierda, agrandamiento del seno cavernoso izquierdo y discreta proptosis del globo ocular izquierdo. La ecografía Doppler presentó dilatación de la vena oftálmica posterior izquierda. Con lo cual se concluyó el diagnostico Fístula Carótido Cavernosa espontánea.

Se controló de la presión arterial con una dosis repetida de Losartan 50mg y de la Presión Intraocular con Acetazolamida 1 comprimido cada 8 horas. Se continuó asociando pilocarpina 1 comprimido cada 6 horas.

Se refirió a servicio hemodinamia en donde se trató con embolización trasvenosa trasfemoral retrógrada, hacia senos cavernosos combinado con la ruta arterial transfemoral anterógrada hacia la carotida cavernosa. 


\section{Examen Físico Globo Ocular}

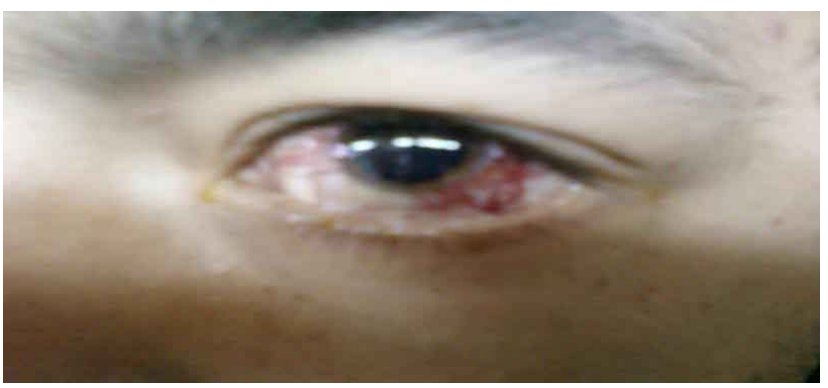

Fig. 1.

Fuente: Cortesía del Hospital Fiorito - Buenos Aires, Argentina. Presencia de Exoftalmia izquierda, quemosis Párpado izquierdo: Edematizado. Conjuntivas: Inflamación conjuntival, presencia de vasos episclerales (cabeza de medusa).

\section{Tomografía Axial Computarizada(TAC)}

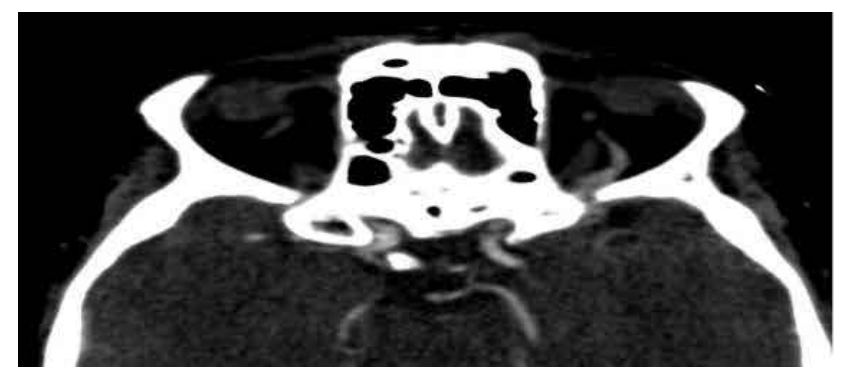

Fig. 2.

Fuente: : Cortesía del Hospital Fiorito - Buenos Aires, Argentina Dilatación de la vena Oftálmica Posterior Izquierda

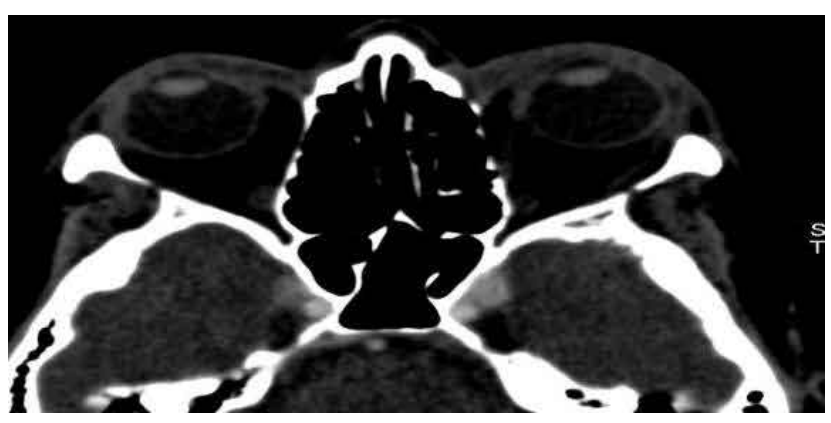

Fig. 3 .

Fuente: : Cortesía del Hospital Fiorito - Buenos Aires Argentina Discreta Proptosis Globo Ocular Izquierdo

\section{Evolución}

A las 72 horas post tratamiento endovascular, se evidenció regresión de la sintomatología incluyendo la disminución de vasos episclerales (Cabeza de Medusa) en globo ocular izquierdo, ausencia de movimientos dolorosos oculares y soplo. Se realizó control de fondo de ojo en el cual se evidencia PIO de $17 \mathrm{mmHg}$. Alta médica a los 5 días, se seguirá un seguimiento con Eco Doppler a los 6 meses para control.

\section{Discusión}

La sintomatología y presencia de signos oculares de las fístulas carótido cavernosas son múltiples y se generan debido a la congestión venosa orbitaria.

Se presenta el caso de un paciente de 53 años de edad masculino, sin antecedentes de traumatismos. Se realizó exploración física oftalmológica, en donde se encontró la tríada clásica descrita por Blanco Cabellos et al. que consta de exoftalmos pulsátil, quemosis y soplo orbitario izquierdo en el globo ocular izquierdo, sin afección del derecho.

Por lo cual se procedió a la realización de una serie de exámenes complementarios, los cuales concuerdan con los estudios basados en reportes del diagnóstico y manejo de Fístula Carótido Cavernosa como el del caso reportado en la revista peruana de neuro-psiquatría publicado en el 2017, que concluye que para el diagnóstico, la Tomografía Computada(TC) y la Resonancia Magnética(RM) cerebrales y de órbitas pueden ser útiles en la detección de signos indirectos o de signos compatibles.

Se encontró como resultado en la TC la presencia de dilatación de la vena Oftálmica Posterior Izquierda y una discreta proptosis en el globo ocular izquierdo.

Belhachmi et al. en el artículo publicado en abril del 2017, en un reporte de caso sobre un paciente con fístula carótido cavernosa de origen espontáneo, como en el caso del paciente de este estudio, con igual presentación clínica, cita que el correcto diagnóstico se evoca en los signos oftálmicos y orbitales, sumada la monitorización diaria mediante Doppler, lo que permite una detección precoz y así proseguir a estudios más específicos. ${ }^{5}$

En el paciente se incluyeron exámenes adicionales de manera no invasiva. Las pruebas de imagen en auge incluyen angiografía por tomografía computarizada no invasiva o angiografía por resonancia magnética. ${ }^{11} \mathrm{MD}$. Henderson and NR Miller en Estados Unidos en el año 2017 muestra un estudio de 53 pacientes con fistula carotideo cavernosa directos, evaluadas con ambas técnicas angiográficas en las cuales se encontró que no difirió la una sobre la otra, manteniendo una sensibilidad entre el 87 vs $94.4 \%$ respectivamente. ${ }^{11}$ Por lo cual se puede concluir que el uso de una de estas dos técnicas podría haber sido beneficioso para un diagnóstico más precoz, aunque si bien es cierto, el pronóstico del paciente fue bueno, con el uso de técnicas más avanzadas el tratamiento hubiera sido más eficaz.

Como se citó anteriormente, el paciente fue tratado de manera clínica para el control de la presión intraocular seguido de tratamiento endovascular con embolización transvenosa como en el caso de la investigación de la revista brasileña de Colegio de Cirujanos, febrero 2017, en el que realizó un estudio retrospectivo se concluyó que tras un diagnóstico precoz, el tratamiento endovascular obtuvo mejores resultados, y se asocia a una menor tasa de morbimortalidad en pacientes con esta patología. ${ }^{12}$ 


\section{Conclusión}

La Fístula Carótido-Cavernosa forma parte de las afecciones neuro-oftálmicas poco frecuentes de tipo espontáneo, pero con una alta incidencia relacionada con traumatismos craneoencefálicos. Dichas fístulas ocurren por varios factores desencadenantes y complicaciones posttraumáticas o espontáneas.

Por ello el conocimiento de las características clínicas y radiológicas junto con un diagnóstico diferencial facilitará el reconocimiento para un correcto diagnóstico precoz y así un tratamiento óptimo para dicha patología.

Como experiencia inicial se sugiere poseer diagnósticos diferenciales, para así poder descartar futuras complicaciones o factores agregados a dicha patología. En el caso del paciente se sugirió primero estabilizar la presión intraocular del ojo izquierdo, ya que cursaba por un cuadro de glaucoma no controlado.

El tratamiento quirúrgico va de la mano con complicaciones debidas a las características anatómicas de esta zona. Por ello la terapia endovascular combinada con una ruta arterial transfemoral se ha sugerido como primera elección en este trastorno, pero sin descartar que en algunos de los casos podría ocurrir un cierre espontáneo del mismo.

\section{Fuente de Financiamiento}

Este estudio es autofinanciado

\section{Conflicto de Intereses}

No existen conflictos personales, profesionales, financie- 1 roso de otro tipo.

\section{Consentimiento Informado}

Los autores cuentan con el consentimiento informado de los paciente para la investigación, la publicación del caso y sus imágenes.

\section{Referencias Bibliográficas}

1. Batún-Garrido J, E HN. Fístula carótido-cavernosa. Hospital Regional de Alta Especialidad. Med Int. Tabasco, Méx; 2014. Available from: http://www.medigraphic. com/pdfs/medintmex/mim-2014/mim145n.pdf.

2. Núñez-Alcántara NV, Ramírez-Quiñones JA, ValenciaChávez AM, Barrientos-Imán DM, Rosa CL, CalderónSanginez JJ, et al. Fistula carótido-cavernosa unilateral con síntomas neuro-oftalmológicos bilaterales e infarto cerebral venoso: Reporte de un caso. Revista de NeuroPsiquiatría. 2017;80(2):137-143.

3. Lang Serrano G. Fistulas carótido cavernosa. Medicina Legal de Costa Rica. 2014 03;31:135 - 139. Available from: http://www.scielo.sa.cr/scielo.php?script=sci_ arttext\&pid=S1409-00152014000100015\&nrm=iso.
4. Ke L, Yang Yn, Yuan J. Bilateral carotid-cavernous fistula with spontaneous resolution: a case report and literature review. Medicine. 2017;96(19).

5. Belhachmi A. Fistules carotido-caverneuses bilatérales spontanées: à propos d'un cas et revue de la littérature. The Pan African Medical Journal. 2017;27.

6. Oumellal J, Bekaert O, Gallas S, Leguerinel C, Palfi S, Derkaoui F, et al. Fistules carotido-caverneuses posttraumatiques à propos d'un cas et revue de la littérature. Pan African Medical Journal. 2015;21(1).

7. Roy AK, Grossberg JA, Osbun JW, Skukalek SL, Howard BM, Ahmad FU, et al. Carotid cavernous fistula after Pipeline placement: a single-center experience and review of the literature. Journal of NeuroInterventional Surgery. 2017;9(2):152-158. Available from: https://jnis.bmj.com/ content/9/2/152.

8. Phan K, Xu J, Leung V, Teng I, Sheik-Ali S, Maharaj M, et al. Orbital approaches for treatment of carotid cavernous fistulas: a systematic review. World neurosurgery. 2016;96:243-251.

9. Chan FH, Shen CY, Liu JT, Li CS. Brainstem Hemorrhage Caused by Direct Carotid-Cavernous Fistula: A Case Report and Literature Review. Interventional Neuroradiology. 2014;20(4):487-494. PMID: 25207913.

10. Estremera-Rodrigo A, Sarasibar-Ezcurra H, AmengualAlemany G, Martín MU. Fístula carótido-cavernosa;.

11. Henderson A, Miller NR. Carotid-cavernous fistula: current concepts in aetiology, investigation, and management. Eye. 2018;32(2):164.

12. Aguiar GBD, Silva JM, Paiva AL, Jory M, Conti ML, Veiga JC. Endovascular treatment of carotid-cavernous vascular lesions. Revista do Colégio Brasileiro de Cirurgiões. 2017;44(1):46-53.

Recibido: 12 de enero de 2018

Aceptado: 5 de febrero de 2019 